\title{
CAPACITY BUILDING FOR HIGH-RESOLUTION LAND COVER INTERCOMPARISON AND VALIDATION: WHAT IS AVAILABLE AND WHAT IS NEEDED
}

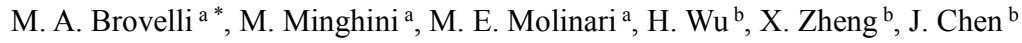 \\ a Department of Civil and Environmental Engineering, Politecnico di Milano, Piazza Leonardo da Vinci 32, 20133 Milano, Italy - \\ (maria.brovelli, marco.minghini, moniaelisa.molinari)@polimi.it \\ ${ }^{\mathrm{b}}$ Department of Remote Sensing and Photogrammetry, National Geomatics Center of China, 28 Lianhuachi West Road, Haidian \\ District, Beijing, 100830, China - (wuhao, xinyan_zheng, chenjun)@ngcc.cn
}

\author{
Commission IV, WG IV/4
}

KEY WORDS: Capacity Building, High-resolution, Intercomparison, Land Cover, Open Source, Training Material, Validation

\begin{abstract}
:
Land Cover (LC) maps are fundamental products for a wide variety of applications. The workflow for their production is composed of classification of satellite imagery and validation against a reference dataset. Different LC maps as well as multiple versions in time of the same LC map can be also compared with one another to assess LC changes. Since the current richness of both space and in-situ observations makes it quite easy to produce LC maps, it is fundamental to assess their accuracy before using them for real applications. This paper focuses on education and capacity building on the intercomparison and validation of global (i.e. covering the whole world) and high-resolution (i.e. with a spatial resolution of at least $30 \mathrm{~m}$ ) LC maps. The availability of Free and Open Source for Geospatial (FOSS4G) technology capable to process LC maps, as well as the existence of ad hoc educational material, is carefully assessed. In parallel, an ad hoc survey has showed that users, especially in developing countries, often lack awareness about the need to validate them and are not aware about the existence of training material. With this premise, a new project presented in the paper aims to produce new, openly licensed and FOSS4G-based training material on the intercomparison and validation of global high-resolution LC maps.
\end{abstract}

\section{INTRODUCTION}

Land Cover (LC) maps are categorical-type products, which describe the nature and characteristics of land surface elements. These maps are fundamental for a wide range of users and applications such as planning, nature and biodiversity protection, economic land use models, natural resources management, hydrological modelling, species distribution modelling, and environmental assessments (see e.g. Foley et al., 2005; Grimm et al., 2008; Nie et al., 2011). LC has also an essential impact on global climate through both biogeochemical and biogeophysical processes (Feddema et al., 2005). LC maps represent a key input to monitor the indicators of the Sustainable Development Goals (SDGs) defined in the 2030 Agenda for Sustainable Development (United Nations, 2015) as well as to promote evidence-based policy-making on issues like soil consumption and deforestation.

LC mapping is one of the earliest applications of Remote Sensing. LC maps usually derive from classification of satellite imagery, i.e. from the conversion of the pixel values in the image into a set of land cover classes. Several classification approaches exist, which are implemented in specialized Remote Sensing software packages. Supervised classification relies on user-selected image pixels representative of the land cover classes selected, which are used to train the classification algorithms to predict the class of all the remaining pixels. In contrast, unsupervised classification algorithms automatically group similar pixels together into unlabeled classes without an a-priori input of information about existing land cover samples (Thomas et al., 1987). In practice, manual approaches (based on the analyst's visual interpretation of satellite imagery) and hybrid approaches, which combine automated, semi-automated and manual classifications, are also adopted to produce higher quality LC maps. Since errors are inherent to the classification, LC maps must undergo an accuracy assessment or validation process to determine their fitness-foruse for specific applications. The validation of a LC map happens by evaluating its degree of agreement with a reference database, considered as the "ground truth" as it indicates the actual LC class for a sample of spatial units, i.e. points, pixels or blocks of pixels (Congalton and Green, 1999). This external database must be generated using a proper probabilistic sample design as an unbiased and representative subset of the population, so that the accuracy assessed for the sample can be used as an estimation of the population's accuracy (Stehman, 2009). Validated LC maps can be compared with each other to assess their mutual similarity. In this case, when none of them is treated as the reference map, they undergone a so-called intercomparison process. This process can happen through the same techniques used for validation, or, in a simpler case, by evaluating the degree of agreement of all the pixels of the two LC maps instead of subsets selected from them. Validation can be also performed using all the pixels - instead of a sample - when the LC map to be assessed is particularly small. Intercomparison between two (or more) successive versions of the same LC map is also applied to study LC evolution over time and detect LC changes.

Following the standard classification and validation workflow, LC maps have been traditionally produced to serve the needs of countries and political organizations. These products show many differences in terms of spatial coverage, spatial resolution, up-todateness and classification scheme. Of particular interest in this paper are global high-resolution LC maps, i.e. those maps having

Corresponding author 
a global coverage and a spatial resolution of at least $30 \mathrm{~m}$. In fact, while several countries and political organizations (e.g. the EU, the USA and Australia) have their own high-resolution LC maps, this is not usually the case for many developing countries, where these maps may be the only available resources of this kind. Thus, it is increasingly important that data users and producers in the fields of Geographic Information Systems (GIS) and Remote Sensing, especially in developing countries, are aware of the existence and importance of global high-resolution LC maps, and able to perform their validation and intercomparison. The ISPRS Education and Capacity Building Initiative named "Capacity Building for High-Resolution Land Cover Intercomparison and Validation" has exactly the purpose of producing openly licensed computer-aided learning material on the intercomparison and validation of high-resolution LC maps based on Free and Open Source Software for Geospatial (FOSS4G). This is preceded by an investigation of the awareness of the importance and needs of LC maps and the requirements towards education in LC maps intercomparison and validation (mainly in developing countries), and the assessment of the currently available training material on the intercomparison and validation of LC maps.

With these premises, the remainder of the paper is structured as follows. Section 2 provides a historical overview of the most important LC mapping projects and related products, with special focus on global and high-resolution maps. A separate review is presented on the use of crowdsourced geographic information for LC mapping purposes, including production and validation. In Section 3 the techniques for validation of LC maps are presented in more detail and a review of intercomparison and validation of existing LC maps is provided. Section 4 presents an overview of FOSS4G packages and related training material to produce and validate LC maps. This is followed by Section 5 , where the first results of the ISPRS-funded project are presented and next steps are outlined. Finally, Section 6 concludes the paper by discussing the main findings and providing directions for future work.

\section{LAND COVER MAPPING PRODUCTS}

At the global level, the earliest LC products were produced at coarse resolutions (between $300 \mathrm{~m}$ and $1 \mathrm{~km}$ ) due to the satellite sensors used and because of their original purpose to serve the needs of the climate modelling community. These maps included: the Global Land Cover 2000 Project (GLC-2000, http://forobs.jrc.ec.europa.eu/products/glc2000/glc2000.php) at $1 \mathrm{~km}$ spatial resolution and classified in 22 classes according to the UN-LCCS scheme (Bartholomé and Belward, 2005); the LC maps derived from MODIS at up to $500 \mathrm{~m}$ spatial resolution and a temporal coverage from 2001 to 2012 (Friedl et al., 2010); the GlobCover map (http://due.esrin.esa.int/page_globcover.php), the first $300 \mathrm{~m}$ global LC map for 2005 (Bontemps et al., 2011); and the European Space Agency (ESA) Land Cover CCI project (https://www.esa-landcover-cci.org/?q=node/1), which produced a 10-year map with three central dates (2000, 2005 and 2010) using the UN-LCCS scheme.

More recently, the opening up of the Landsat archive in 2008 has inaugurated the production of global high-resolution LC products. Examples of global LC maps at $30 \mathrm{~m}$ spatial resolution are the Finer Resolution Observation and Monitoring of Global Land Cover (FROM-GLC, http://data.ess.tsinghua.edu.cn; Gong et al., 2013) and the GlobeLand30 (GL30, http://globeland30.org; Chen et al., 2015). Other datasets were instead produced for specific LC classes, including the $30 \mathrm{~m}$ global surface water from Landsat (Pekel et al., 2016) and the $25 \mathrm{~m}$ global map of forest and nonforest cover from Advanced Land Observing Satellite (ALOS)
Synthetic Aperture Radar (SAR) imagery (Shimada et al., 2014). Two recently released global products aim to differentiate builtup from non built-up areas, i.e. the Global Urban Footprint (GUF) at up to $12 \mathrm{~m}$ resolution (Esch et al., 2013) and the Global Human Settlement Layer (GHSL) (Pesaresi et al., 2016) at $38 \mathrm{~m}$ resolution and available for 1975, 1990, 2000, and 2014. At the European level, the main LC reference maps are CORINE Land Cover (CLC, https://and.copernicus.eu/pan-european/corineland-cover), available for 4 different time periods (1990, 2000, 2006 and 2012) at a spatial resolution up to $100 \mathrm{~m}$; and the Urban Atlas (UA, https://land.copernicus.eu/local/urban-atlas), which provides detailed classification of European urban areas for the reference years 2006 and 2012. Finally, Land Use Cover Area frame Sample (LUCAS, http://ec.europa.eu/eurostat/web/lucas) is the European official LC in-situ dataset, derived from rigorous surveys carried out every 3 years at points systematically located across EU member states (Eurostat, 2015).

The production cycles of LC maps are traditionally long and expensive, as they imply the acquisition of satellite imagery and the need of specialized software and hardware. As a result, these maps are always referred to a specific year or period of time and they quickly become out-of-date. Nowadays, this situation is rapidly changing since many Earth Observation programmes are providing end users with low or no-cost high-resolution imagery on a daily basis. The most ambitious among these programmes is Copernicus (http://copernicus.eu), which exploits data delivered from ESA Sentinel satellites with the overall mission to establish a European capacity for Earth Observation. Land monitoring is one of the core services provided by Copernicus thanks to the Sentinel-2 mission (ESA, 2015), whose satellites acquire up to $10 \mathrm{~m}$ optical imagery with a few days revisiting time. All Sentinel datasets are available for end users under an open access license, thus opening new possibilities in data exploitation for LC mapping purposes. As an example, by taking advantage of 1 year of Sentinel-2 observations (from December 2015 to December 2016), a prototype LC map at $20 \mathrm{~m}$ resolution was developed for the whole Africa (http://2016africalandcover20m.esrin.esa.int).

\subsection{Crowdsourced geographic information for $\mathrm{LC}$}

Crowdsourced geographic information (See et al., 2016) is here used as a general term referring to the provision of geographic information by citizens, a phenomenon that literature describes with a multitude of terms including Volunteered Geographic Information (VGI), Citizen Science, Participatory Sensing, and user-generated content, to name but a few. Several characteristics make citizen-sensed geographic datasets highly suitable for LC mapping, for example their (potential) ubiquity, up-to-dateness, richness and level of detail, which in many cases overcome those of any authoritative product. Several efforts are currently in place to integrate space and volunteered in-situ observations within official projects and programmes. For instance, in the last few years a number of EU projects (so-called Citizen Observatories) have been funded with this exact purpose, including LandSense (https://landsense.eu), SCENT (https://scent-project.eu) and GroundTruth2.0 (http://gt20.eu). Fritz et al. (2017) observe that the primary actions performed by citizens in this context consist of image interpretation and in-situ data collection, both of which are useful for calibrating and validating satellite imagery or derived products such as LC maps. Fonte et al. (2015) review the use of crowdsourced geographic information as reference data for LC map validation and analyse the potentially most useful projects and types of citizen-sensed datasets. Instead, Stehman et al. (2018) discuss the options for incorporating such datasets within design-based inference aimed ad validating LC maps. 
Several projects have the explicit objective of involving citizens in collecting LC data for map training and validation. The most well-established initiative of this kind is Geo-Wiki (Fritz et al., 2009, https://www.geo-wiki.org), which involves the crowd in the classification of satellite imagery (e.g. Google Earth) to produce datasets for calibration and validation of LC maps. The project was started to study the spatial disagreement between the global LC maps GLC-2000, MODIS and GlobCover, and offers targeted classifications campaigns on some LC classes such as croplands (Salk et al., 2016). A similar approach is adopted in the Virtual Interpretation of Earth Web-Interface Tool (VIEW-IT), whose purpose is to produce LC maps and verify their accuracy based on volunteers' interpretation of reference data from Google Earth imagery (Clark and Aide, 2011). Brovelli et al. (2018) organized a game-based crowdsourcing campaign of the same kind to evaluate the disagreement between the GL30 and a local LC map in Como, northern Italy. In other cases data collection happens through field activities. This is e.g. the case of FotoQuest Austria, a mobile app created within Geo-Wiki, which involves citizens in classifying land use/cover at the same locations of LUCAS sample points in Austria (Laso Bayas et al., 2016).

In several other crowdsourcing projects, data is gathered for other purposes but may be very useful for LC mapping. Examples are platforms for sharing geotagged photographs, such as landscapeoriented initiatives like Geograph (https://www.geograph.org) and the Degree Confluence Project (http://confluence.org), or more general-purpose sites like Flickr (https://www.flickr.com), Instagram (https://www.instagram.com) and Google's Panoramio (offline since November 2016). These open repositories have shown great potential for training, validation and verification of LC maps, with the most promising results obtained by landscape-oriented initiatives (Antoniou et al., 2016). However, the crowd-based initiative having the greatest potential for LC mapping is OpenStreetMap (OSM; Mooney and Minghini, 2017), which has produced the largest, most detailed, up-to-date, and complete geospatial database of the whole world. Jokar Arsanjani et al. (2013) converted OSM into a LC map with the UA nomenclature in Vienna, Austria, finding positive results from the comparison. Fonte et al. (2017a) developed an automated methodology for converting OSM into the LC classes of UA. Deriving from OSM, these LC maps usually suffer from LC data gaps in regions where OSM is absent. To overcome this issue, Fonte et al. (2017b) superimposed a detailed and up-to-date LC map derived from OSM to GL30, resulting in an updated version of the latter. Instead, Schultz et al. (2017) exploited OSM to derive a LC map compliant to the nomenclature of CLC (http://osmlanduse.org) and filled the LC gaps through a supervised classification using selected OSM features as training areas.

\section{LC MAPS INTERCOMPARISON AND VALIDATION}

Since the mid-1980s, the confusion (or error) matrix represents the standard approach for the validation and intercomparison of remotely sensed data (Congalton and Green, 1999). According to this approach, the validation of a LC map against a reference database - or the comparison with another LC map - happens by building a matrix, in which agreements and disagreements in thematic classification between the two datasets are computed. Multiple accuracy measures can be extracted from a confusion matrix to assess the quality of the LC product under evaluation, both globally and for the single LC classes. The most commonly used indexes are overall accuracy, user's accuracy and producer's accuracy. Many other measures were also proposed in addition to these. Strictly related to the previous indexes are the average of the user's and producer's accuracies (Fung and LeDrew, 1988), the Hellden's mean accuracy (Liu, 2007) and the Individual Classification Success Index (ICSI; Koukoulas and Blackburn, 2001). Other measures are grouped into the so-called kappa-like statistics, which include standard kappa, conditional kappa and weighted kappa (Cohen, 1968), Tau index of agreement (Ma and Redmond, 1995), Aickin's alfa (Aickin, 1990) and Ground Truth Index (Türk, 1979). Instead, the Average Mutual Information (AMI) is derived from the information theory (Abramson, 1963) and a Normalized Mutual Information (NMI) is proposed by Finn (1993). Finally, indexes for computation of measures related to disagreement and its allocation and quantity components are proposed by Pontius and Millones (2011).

Validation of available high-resolution LC maps (see Section 2) is still undergoing at the international level to determine their usability for different applications. For example, Brovelli et al. (2015) performed the first accuracy assessment of the GL30 at the national level through comparison with more detailed LC datasets available in Italy, finding overall accuracy values higher than $80 \%$. The validation exercise was repeated in Germany by Jokar Arsanjani et al. (2016), who compared GL30 with existing datasets (CLC, UA, OSM and the national ATKIS) finding high agreement up to $92 \%$. An overall accuracy of $91.9 \%$ was obtained by Manakos et al. (2015) when validating the land surface water and the drainage network layers of GL30 for a region in Greece through comparison with ground truth data from the Hellenic Cadastre. Jokar Arsanjani (2018) performed an intercomparison between the 2000 and 2010 versions of GL30 at a global level, highlighting massive LC change patterns such as deforestation, desertification, urbanization and shrinkage of water bodies. The new GUF and GHSL products have also attracted the interest of researchers, who started to assess their accuracy. For example, Minghini et al. (2017) compared GUF and GHSL both with each other and with official products (UA and LUCAS) for Milan, Italy. GUF and GHSL were found to be very similar to each other and, with some exceptions, they showed overall good agreements with the reference LC datasets. Using appropriate reference data, Mück et al. (2017) validated GUF for Burkina Faso, showing its enhanced mapping capabilities for rural areas. Leyk et al. (2018) developed an accuracy assessment framework for multi-temporal built-up layers using public parcels and building records as validation data. The framework was successfully tested on GHSL in the Unites States.

\section{FOSS4G FOR LAND COVER MAPPING}

Brovelli et al. (2017) recently assessed the reliability and maturity of FOSS4G technology to address the needs and demands of current society. The authors show many examples - among others - of how FOSS4G solutions are implemented in practice towards modelling Earth systems and human dynamics and contribute to sustainable development. Indeed the FOSS4G ecosystem, meant as the combination of the software itself and the corresponding community (developers, users, educators, etc.), offers valuable resources in the field of production and validation of LC maps which is of main interest in this work. An overview is provided in the following on the most relevant FOSS4G packages for LC mapping (including specific modules or extensions) and related learning material, where available. These serve as a starting point for the Capacity Building Initiative described in Section 5.

\subsection{QGIS}

QGIS (https://qgis.org) is the leading desktop GIS software in the FOSS4G arena. It is an official project of the Open Source 
Geospatial Foundation (OSGeo, https://www.osgeo.org) and it is available under the GNU General Public License (GPL) (Free Software Foundation, 2007). The latest stable QGIS version is 3.0, released in early 2018. QGIS provides an intuitive and easyto-use interface to visualise, edit, analyse, process and publish geospatial data. One of the main reasons behind the success of QGIS lies in its modular architecture composed of plugins, i.e. software extensions (relatively easy to be developed) focused on performing specific tasks. The most significant tools provided by QGIS to handle LC maps are exactly available into dedicated plugins, as described in the following.

4.1.1 Semi-Automatic Classification Plugin (SCP): a QGIS plugin featuring advanced tools for download, preprocessing and postprocessing of imagery by ASTER, Landsat, MODIS and Sentinel-2 (https://fromgistors.blogspot.com/p/semi-automaticclassification-plugin.html). In particular, the plugin offers several algorithms for the supervised and unsupervised classification of satellite imagery. The SCP is available for QGIS 2 and has been recently rewritten for QGIS 3; the source code is available at https://github.com/semiautomaticgit/SemiAutomaticClassificati onPlugin under the GNU GPL (Free Software Foundation, 2007). In addition to an introduction to Remote Sensing and the plugin interface, the documentation (Congedo, 2016) includes a step-bystep tutorial on how to perform land cover classification (see $\mathrm{http}: / /$ semiautomaticclassificationmanual.readthedocs.io/en/mas ter/tutorial_1.html). Validation and intercomparison of LC maps are also possible using the plugin, which returns the confusion matrix and the related metrics of overall accuracy, producer's accuracy, user's accuracy, kappa coefficient and its variance. Learning material about validation is also available, for instance the tutorial at https://fromgistors.blogspot.com/2014/01/landcover-classification-of-cropland.html. This comprehensive set of tutorials, available as web pages under the CC BY-SA 4.0 license (Creative Commons, 2018), is enriched by videos (all published on YouTube) and screenshots (see e.g. Figure 1).

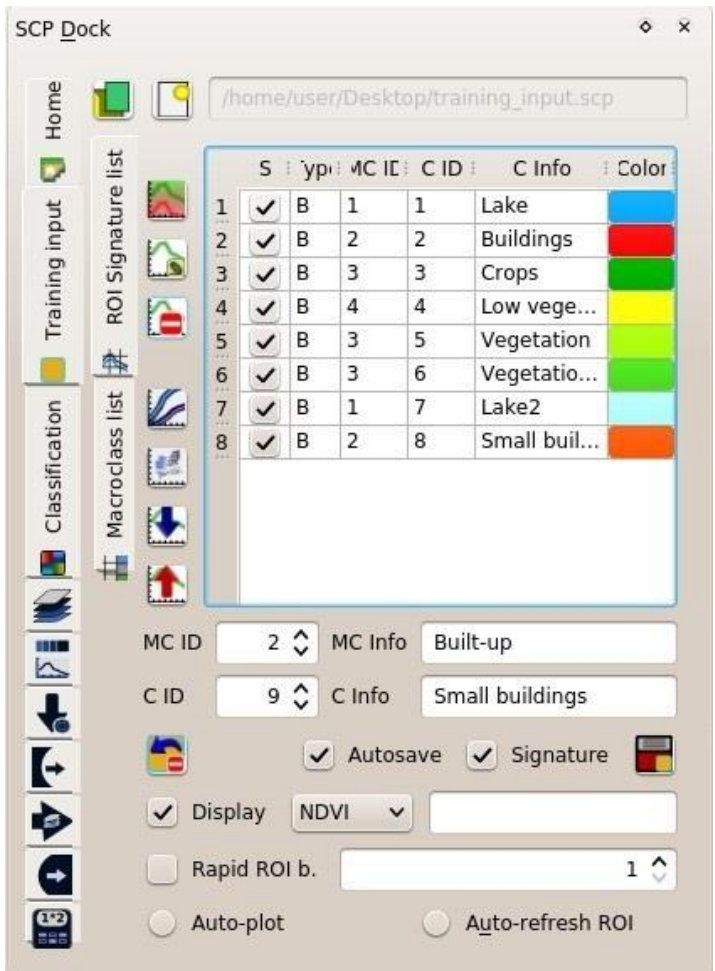

Figure 1. Definition of LC class colors using the SCP. Source: http://semiautomaticclassificationmanual.readthedocs.io/en/mas ter/tutorial_1.html.
Accuracy Assessment plugin: a plugin able to compare two LC raster maps available in QGIS and output a confusion matrix as a Comma Separated Values (CSV) file, which can then be loaded into a spreadsheet to allow for additional analysis and processing (https://plugins.qgis.org/plugins/accassess). The plugin simply requires to select the reference and the comparison LC maps and the name and path of the output file (see Figure 2). The source code is available at https://github.com/jkibele/acc-assess under the BSD-3-Clause license (SPDX, 2018). The plugin is written in Python and is only available for QGIS 2, since its development apparently stopped in 2014. Apart from a short explanation of the plugin's output included in the GitHub repository README file, no tutorial or other learning material was found.

\begin{tabular}{|c|c|}
\hline AccAssess & $-+x$ \\
\hline \multicolumn{2}{|l|}{ Reference Map } \\
\hline GL30 & $\because$ \\
\hline \multicolumn{2}{|l|}{ Comparison Map } \\
\hline LC map from Sentinel-2 & $\div$ \\
\hline \multicolumn{2}{|l|}{ CSV Output File } \\
\hline ne/user/confusion_matrix.csv & Select... \\
\hline Cancel & $\underline{\mathrm{OK}}$ \\
\hline
\end{tabular}

Figure 2. Interface of the QGIS Accuracy Assessment plugin.

\subsection{GRASS GIS}

Geographical Resources Analysis Support System (GRASS) GIS (https://grass.osgeo.org) is a software suite suitable for advanced management and analysis of geospatial data, image processing, visualization, spatial modelling, graphics and maps production. It is licensed under the GPL (Free Software Foundation, 2007) and is an official project of OSGeo. GRASS GIS offers a modular architecture to help users navigate into the wealth of available tools (Neteler et al., 2012). Modules are organized by category (e.g. vector, raster, database, image, etc.) and by function (input, output, etc.). The relevant GRASS modules for managing LC maps belong to the modules for image data and raster data.

Image classification is mainly achieved using the module i.maxlik (https://grass.osgeo.org/grass74/manuals/i.maxlik.html), which makes use of the spectral signature data generated by i.cluster (https://grass.osgeo.org/grass 74/manuals/i.cluster.html) and i.gensig (https://grass.osgeo.org/grass74/manuals/i.gensig.html) for unsupervised and supervised classification, respectively. The g.gui.iclass module is instead a classification tool that generates spectral signatures by allowing users to outline regions of interest (https://grass.osgeo.org/grass74/manuals/g.gui.iclass.html). In terms of validation of LC products, the main GRASS GIS module is r.kappa (https://grass.osgeo.org/grass 74/manuals/r.kappa.html, see Figure 3), which computes the confusion matrix and returns accuracy measures such as overall kappa, conditional kappa, overall accuracy, user's accuracy and producer's accuracy. A useful module is also r.reclass, allowing to reclassify a raster map based on the LC classes of a target raster map used for validation (https://grass.osgeo.org/grass74/manuals/r.reclass.html). GRASS GIS reference manual (https://grass.osgeo.org/grass74/manuals), which details the use of each module, is itself a source of learning material. The section of the manual related to image processing, 
which includes both image classification and validation, provides a more compact list of the relevant modules mentioned above (https:/grass.osgeo.org/grass74/manuals/imageryintro.html). A short tutorial on image classification in GRASS GIS is available at https://grasswiki.osgeo.org/wiki/Image_classification.

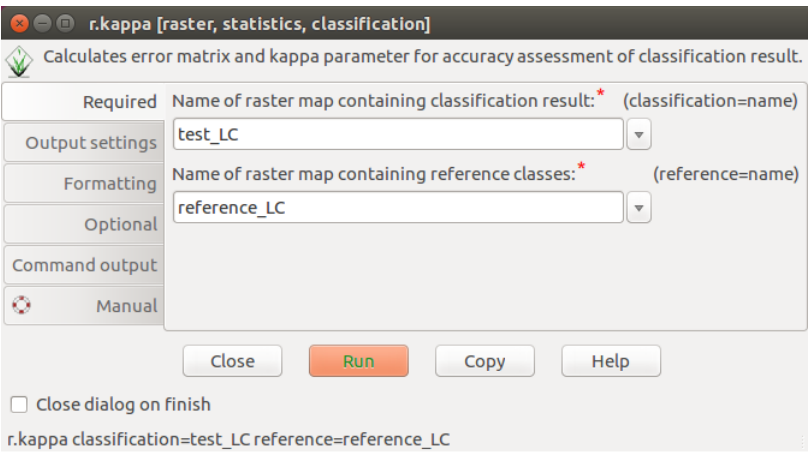

Figure 3. Interface of the GRASS GIS module r.kappa.

\subsection{Orfeo ToolBox}

Orfeo ToolBox (OTB, https://www.orfeo-toolbox.org) is an open source project providing state-of-the-art processing functions for remotely-sensed imagery. All its algorithms are accessible from other FOSS4G technology such as Monteverdi (an image viewer, https://www.orfeo-toolbox.org/CookBook/Monteverdi.html) and QGIS, as well as from Python, $\mathrm{C}++$ and the command line. OTB is an official project of OSGeo, is licensed under the Apache License 2.0 (Apache Software Foundation, 2004) and source code is hosted at https://gitlab.orfeo-toolbox.org/orfeotoolbox/otb. Being specifically developed for Remote Sensing purposes, OTB offers algorithms for unsupervised and supervised classification provided by the TrainVectorClassifier (https://www.orfeotoolbox.org/CookBook/Applications/app_TrainVectorClassifier. html) and the TrainImagesClassifier (https://www.orfeotoolbox.org/CookBook/Applications/app TrainImagesClassifier .html) applications. It also provides validation capabilities thanks to the ComputeConfusionMatrix application (https://www.orfeotoolbox.org/CookBook/Applications/app_ComputeConfusionM atrix.html), which returns the confusion matrix in CSV format together with the overall accuracy and the user's and producer's accuracy for each LC class. A set of guidelines (named "recipes") on how to use OTB algorithms are contained in the OTB website, including one on classification and validation: https://www.orfeotoolbox.org/CookBook/recipes/pbclassif.html. A tutorial on how to perform supervised classification with OTB is available at http://gracilis.carleton.ca/CUOSGwiki/index.php/Image_Classif ication_Tutorial_using_Orfeo_Toolbox.

\subsection{SAGA}

Acronym for System for Automated Geoscientific Analyses, SAGA (http://www.saga-gis.org) is a comprehensive platform for scientific analysis and modelling. It features a modular software architecture with hundreds of available tools suitable for a wide spectrum of scientific applications (Conrad et al., 2015). SAGA source code is hosted at https://sourceforge.net/p/saga-gis/code and it is in large part released under the GNU GPL (Free Software Foundation, 2007). SAGA offers a dedicated API, which makes its algorithms accessible through many scripting methods; in the same way as OTB, these algorithms are also available in QGIS. The library documentation of SAGA lists 7 tools for performing classification of imagery (both supervised and unsupervised) and validating the classification results through the creation of a confusion matrix and the derived coefficients (http://www.sagagis.org/saga tool doc/6.3.0/imagery classification.html). A nice set of tutorial is available on the use of SAGA for LC mapping (https://sagatutorials.wordpress.com). They include tutorials on both supervised and unsupervised imagery classification (see $\mathrm{https}$ ://sagatutorials.wordpress.com/unsupervised-classification, https://sagatutorials.wordpress.com/supervised-segmentationclassification) and a tutorial on LC change mapping, which features both the steps of classification and intercomparison: https://sagatutorials.wordpress.com/land-cover-change-mapping.

\subsection{SNAP}

Sentinel Application Platform (SNAP, http://step.esa.int/main) is a dedicated open source toolbox developed by ESA for analysing and processing products from Sentinel 1,2 and 3. SNAP is licensed under the GNU GPL (Free Software Foundation, 2007) and the source code is available at https://github.com/senbox-org. Sentinel-2 toolbox (http://step.esa.int/main/toolboxes/sentinel-2toolbox) offers a rich set of tools for the visualisation, analysis and processing of high-resolution optical imagery. These include algorithms for both supervised and unsupervised classification, however neither an official user guide nor specific tutorials or learning materials were found. Some references are reported in a forum discussion at $\mathrm{http}: / /$ forum.step.esa.int/t/supervised-andunsupervised-classification-sentinel-2/3388. Also, validation or intercomparison tools are not available in SNAP. Despite the absence of a SNAP user guide, it is worth noticing that ESA is regularly organizing dedicated training events on land Remote Sensing topics, see e.g. http://eoscience.esa.int/landtraining2017 and http://eoscience.esa.int/landtraining2018.

\section{CAPACITY BUILDING ON LC MAPPING}

In line with its mission of strengthening educational activities, the International Society for Photogrammetry and Remote Sensing (ISPRS) has recently funded the project "Capacity Building for High-Resolution Land Cover Intercomparison and Validation" within the series of Educational and Capacity Building Initiatives 2018 (http://www.isprs.org/society/ecbi/default.aspx). The goal of the project is to create new knowledge and tools to educate and raise awareness on the intercomparison and validation of global high-resolution LC maps, mainly in developing countries. More in detail, the initiative - started in February 2018 and ending in January 2019 - is composed of the following four tasks:

- analysis of the needs, requirements and limiting factors in using and validating LC maps from a user's perspective, with special focus on developing countries;

- assessment and classification of the available training material on intercomparison and validation of global high-resolution LC maps;

- development of new computer aided educational material on the intercomparison and validation of global high-resolution LC maps. This will include both teaching material (e.g. slide presentations and text documents), released under open access licenses, and software-based material (e.g. new modules for GIS software and code scripts/repositories), released under open source licenses to maximize the exploitation and impact within the community;

- organization of three workshops on the intercomparison and validation of global high-resolution LC maps, two of which held in developing countries (Dar es Salaam in Tanzania and Nairobi in Kenya).

At the time of writing (April 2018) the first and second tasks are taking place. The first task is mainly accomplished through an ad hoc questionnaire aimed at assessing the general awareness about the existence and importance of LC maps as well as the need for 
their intercomparison and validation. Respondents are also asked to list which LC maps they have used, for which applications, and which is the resolution of LC maps that they believe is useful for these specific applications. The questionnaire is available at https://tinyurl.com/ydgg59ua. Partial results extracted from the answers to this questionnaire, which is still open for submission, show that most of the respondents are familiar with LC maps, have already used them for research and/or professional works, and consider them to be of primary importance for their specific applications. However, only about $1 / 3$ of the respondents are fully aware about the importance of the LC validation process, mentioning applications such as economic land use modelling, flood modelling, land management, land degradation assessment, large scale policy and decision making. In terms of the LC maps already used, most of the respondents listed products derived from Landsat, MODIS, and, to a lesser extent, Sentinel imagery. Some European respondents also indicated the CLC and UA LC maps, while GL30 was only mentioned by few respondents. In one single case, LC maps derived from OSM were also cited. Most of the respondents agreed that the ideal resolution of LC maps depends on the specific needs of the application where they are used, however the benefits of high-resolution maps (i.e. with a spatial resolution of at least $30 \mathrm{~m}$ ) were also highlighted.

The second task of the project, i.e. assessing the available training material on the intercomparison and validation of global highresolution LC maps, was performed by coupling a manual review of the state of the art (to which Section 4 of this paper, focused on FOSS4G, contributes) with a second questionnaire, available at https://tinyurl.com/yde9ykqg. This questionnaire simply asks respondents to indicate their knowledge of training or educational material, in particular: title/name, type (text document, slides, web application, etc.), reference software used, license, objective of the training and target beneficiaries, LC maps used within the material, and link to the material (if available). This questionnaire is also still open for submission, however at the time of writing it has received only around $2 / 3$ of the answers compared to the first questionnaire. Partial results highlight a heterogeneous picture in terms of the type of material (mainly web applications, websites, slides and documents) and software used, with answers almost equally distributed between proprietary software (ArcGIS, ENVI, Google Earth Engine, eCognition and ERDAS IMAGINE) and open source software (QGIS and GRASS GIS). The beneficiaries of the training material are primarily (i.e. for half of the submitted answers) students, followed by researchers and university staff, governmental agencies, professionals and software developers. Less than half of the respondents provided a link to the training material mentioned in their submission.

Both the questionnaires were distributed among several thematic, research as well as educational networks, including GeoForAll (https://www.osgeo.org/initiatives/geo-for-all), i.e. the OSGeo's Committee for educational outreach. Other networks specifically focused on developing countries were also addressed: these include YouthMappers (http://www.youthmappers.org) and the Humanitarian OpenStreetMap Team (https://www.hotosm.org). At the time of writing, almost two thirds of the answers came from people working in developing countries, thus confirming that the main target group of the project was reached. Based on the final results of the questionnaires and, in particular, the most relevant use cases identified for the use of LC maps, the third and fourth tasks of the project will be accomplished through the creation of ad hoc training material and the related software, as well as their practical tests during the provision of workshops. As global highresolution LC map to be used for the training, the choice will most probably fall on the GL30, which, in addition to the chance of validation against local LC products, features the two versions from 2000 and 2010 that can be compared to assess LC changes.

\section{CONCLUSIONS}

LC maps represent key information for a variety of applications. The non-stop developments in space technology and the success of crowdsourcing projects currently produce a rich and constant amount of data (space and in-situ observations, respectively) with great potential for LC-related purposes. The combination of these two types of data is also opening new and interesting possibilities (Fritz et al., 2017). However, if on one side there is an increased availability of LC maps, on the other side it is fundamental that their usage is preceded by a proper quality assessment. This paper has addressed the problem of education and capacity building on the intercomparison and validation of global high-resolution LC maps, focusing in particular on the breadth of available FOSS4G technology and the related educational material. The analysis has shown a rich and heterogeneous picture, with many software packages existing, which provide functionality for classification and validation of LC maps. Available training material usually consists of the user manual of the software itself, with only few cases when ad hoc tutorials are created to address the specific need of classifying or validating a LC product. Thus, this material is often very technical and may remain the sole prerogative of the user community of one particular software. For all these reasons, raising awareness on the importance of LC maps and building capacity on how to compare or validate them represents an asset for students, researchers, professionals and all users of LC data in the domains of GIS and Remote Sensing. A recently started project, which was briefly presented in the paper, has showed that while users generally recognize the importance of LC maps, they often lack awareness about the need to validate them at the local scale and are not aware about ad hoc training material. Thus, the project will bring an important educational contribution to this domain, with special focus on developing countries. In line with the spirit of open science, the developed training material will be released as open access and will be fully based on FOSS4G, which, as showed in the paper, constitutes mature, efficient and reliable technology.

\section{ACKNOWLEDGEMENTS}

This work is partially funded by the ISPRS 2018 Education and Capacity Building Initiative named "Capacity Building for HighResolution Land Cover Inter-comparison and Validation".

\section{REFERENCES}

Abramson, N., 1963. Information theory and coding. McGrawHill, New York.

Aickin, M., 1990. Maximum Likelihood Estimation of Agreement in the Constant Predictive Probability Model and Its Relation to Cohen's Kappa. Biometrics, 46(2), pp. 293-302.

Antoniou, V., Fonte, C.C., See, L., Estima, J., Jokar Arsanjani, J., Lupia, F., Minghini, M., Foody, G.M., and Fritz, S., 2016. Investigating the feasibility of geo-tagged photographs as sources of land cover input data. ISPRS International Journal of GeoInformation, 5(5), 64.

Apache Software Foundation, 2004. Apache License Version 2.0. https://www.apache.org/licenses/LICENSE-2.0 (30 April 2018). 
Bartholomé, E., and Belward, A.S., 2005. GLC2000: a new approach to global land cover mapping from Earth observation data. International Journal of Remote Sensing, 26(9), pp. 19591977.

Bontemps, S., Defourny, P., Van Bogaert, E., Arino, O., Kalogirou, V., and Perez, J.R., 2011. GLOBCOVER 2009 Products Description and Validation Report.

Brovelli M.A., Molinari M.E., Hussein E., Chen, J., and Li, R., 2015. The first comprehensive accuracy assessment of GlobeLand30 at a national level: Methodology and results. Remote Sensing, 7(4), pp. 4191-4212.

Brovelli, M.A., Minghini, M., Moreno-Sanchez, R., and Oliveira, R. 2017. Free and open source software for geospatial applications (FOSS4G) to support Future Earth. International Journal of Digital Earth, 10(4), pp. 386-404.

Brovelli, M.A., Mooney, P., Biagi, L., Brambilla, M., Celino, I., Ciceri, E., Dorigatti, N., Huang, H., Minghini, M., and Venkatachalam, V., 2018. Mapping Parties at FOSS4G Europe: Fun, Outcomes and Lessons Learned. In: Mobile Information Systems Leveraging Volunteered Geographic Information for Earth Observation. Springer, Cham, pp. 3-34.

Chen, J., Chen, J., Liao, A., Cao, X., Chen, L., Chen, X., He, C., Han, G., Peng, S., Lu, M., Zhang, W., Tong, X., and Mills, J., 2015. Global land cover mapping at $30 \mathrm{~m}$ resolution: A POKbased operational approach. ISPRS Journal of Photogrammetry and Remote Sensing, 103, pp. 7-27.

Clark, M.L., and Aide, T.M., 2011. Virtual Interpretation of Earth Web-Interface Tool (VIEW-IT) for collecting land-use/landcover reference data. Remote Sensing, 2011(3), pp. 601-620.

Cohen, J., 1968. Weighted kappa: Nominal scale agreement provision for scaled disagreement or partial credit. Psychological Bulletin, 70(4), pp. 213-220.

Congalton, R.G., and Green, K., 1999. Assessing the Accuracy of Remotely Sensed Data: Principles and Practices. CRC Press, Boca Raton.

Congedo, L., 2016. Semi-Automatic Classification Plugin Documentation.

http://semiautomaticclassificationmanual.readthedocs.io/en/mas ter/index.html (30 April 2018).

Conrad, O., Bechtel, B., Bock, M., Dietrich, H., Fischer, E., Gerlitz, L., Wehberg, J., Wichmann, V., and Böhner, J., 2015. System for Automated Geoscientific Analyses (SAGA) v. 2.1. 4. Geoscientific Model Development, 8(7), pp. 1991-2007.

Creative Commons, 2018. Attribution-ShareAlike 4.0 International (CC $\quad$ BY-SA 4.0$)$ https://creativecommons.org/licenses/by-sa/4.0 (30 April 2018).

ESA, 2015. SENTINEL-2 User Handbook. https://sentinel.esa.int/documents/247904/685211/Sentinel-

2_User_Handbook (30 April 2018).

Esch, T., Marconcini, M., Felbier, A., Roth, A., Heldens, W., Huber, M., Schwinger, M., Taubenböck, H., Müller, A., and Dech, S., 2013. Urban Footprint Processor-Fully Automated Processing Chain Generating Settlement Masks From Global Data of the TanDEM-X Mission. IEEE Geoscience and Remote Sensing Letters, 10(6), pp. 1617-1621.
Eurostat, 2015. LUCAS 2015 (Land Use / Cover Area Frame Survey). Technical reference document $\mathrm{C} 1$ Instructions for Surveyors.

http://ec.europa.eu/eurostat/documents/205002/6786255/LUCA S2015-C1-Instructions-20150227.pdf (30 April 2018).

Feddema, J.J., Oleson, K.W., Bonan, G.B., Mearns, L.O., Buja, L.E., Meehl, G.A., and Washington, W.M., 2005. The importance of land-cover change in simulating future climates. Science, 310(5754), pp. 1674-1678.

Finn, J.T., 1993. Use of the average mutual information index in evaluating classification error and consistency. International Journal of Geographical Information Science, 7(4), pp. 349-366.

Foley, J.A., DeFries, R., Asner, G.P., Barford, C., Bonan, G., Carpenter, S.R., Chapin, F.S., Coe, M.T., Daily, G.C., Gibbs, H.K., Helkowski, J.H., Holloway, T., Howard, E.A., Kucharik, C.J., Monfreda, C., Patz, J.A., Prentice, I.C., Ramankutty, N., and Snyder, P.K., 2005. Global Consequences of Land Use. Science, 309(5734), pp. 570-574

Fonte, C.C., Bastin, L., See, L., Foody, G.M., and Lupia, F., 2015. Usability of VGI for validation of land cover maps. International Journal of Geographical Information Science, 29(7), pp. 12691291.

Fonte, C.C., Patriarca, J.A., Minghini, M., Antoniou, V., See, L., and Brovelli, M.A., 2017a. Using OpenStreetMap to Create Land Use and Land Cover Maps: Development of an Application. In: Volunteered Geographic Information and the Future of Geospatial Data. IGI Global, Hershey, pp. 113-137.

Fonte C.C., Minghini, M., Patriarca, J., Antoniou, V., See, L., and Skopeliti, A., 2017b. Generating up-to-date and detailed land use and land cover maps using OpenStreetMap and GlobeLand30. ISPRS International Journal of Geo-Information, 6(4), 125.

Free Software Foundation, 2007. GNU General Public License, https://www.gnu.org/licenses/gpl-3.0.en.html (30 April 2018).

Friedl, M.A., Sulla-Menashe, D., Tan, B., Schneider, A., Ramankutty, N., Sibley, A., and Huang, X., 2010. MODIS Collection 5 global land cover: Algorithm refinements and characterization of new datasets. Remote Sensing of Environment, 114(1), pp. 168-182.

Fritz, S., McCallum, I., Schill, C., Perger, C., and Grillmayer, R., 2009. Geo-Wiki.Org: The use of crowdsourcing to improve global land cover. Remote Sensing, 1(3), pp. 345-354.

Fritz, S., Fonte, C.C., and See, L., 2017. The Role of Citizen Science in Earth Observation. Remote Sensing, 9(4), 357.

Fung, T., and LeDrew, E., 1988. The Determination of Optimal Threshold Levels for Change Detection Using Various Accuracy Indices. Photogrammetric Engineering and Remote Sensing, 54(10), pp. 1449-1454.

Gong, P., Wang, J., Yu, L., Zhao, Y.C., Zhao, Y.Y., Liang, L., Niu, Z.G., Huang, X.M., Fu, H.H., Liu, S., Li, C.C., Li, X.Y., Fu, W., Liu, C.X., Xu, Y., Wang, X.Y., Cheng, Q., Hu, L.Y., Yao, W.B., Zhang, H., Zhu, P., Zhao, Z.Y., Zhang, H.Y., Zheng, Y.M., Ji, L.Y., Zhang, Y.W., Chen, H., Yan, A., Guo, J.H., Yu, L., Wang, L., Liu, X.J., Shi, T.T., Zhu, M.H., Chen, Y.L., Yang, G.W., Tang, P., Xu, B., Ciri, C., Clinton, N., Zhu, Z.L., Chen, J., and Chen, J., 2013. Finer resolution observation and monitoring of global land 
cover: first mapping results with Landsat TM and ETM+ data. International Journal of Remote Sensing, 34(7), pp. 2607-2654.

Grimm, N.B., Faeth, S.H., Golubiewski, N.E., Redman, C.L., Wu, J., Bai, X., and Briggs, J.M., 2008. Global change and the ecology of cities. Science, 319(5864), pp. 756-760.

Jokar Arsanjani, J., Helbich, M., Bakillah, M., Hagenauer, J., and Zipf, A., 2013. Toward mapping land-use patterns from volunteered geographic information. International Journal of Geographical Information Science, 27(12), pp. 2264-2278.

Jokar Arsanjani, J., See, L., and Tayyebi, A., 2016. Assessing the suitability of GlobeLand30 for mapping land cover in Germany. International Journal of Digital Earth, 9(9), pp. 873-891.

Jokar Arsanjani, J., 2018. Characterizing and monitoring global landscapes using GlobeLand30 datasets: the first decade of the twenty-first century. International Journal of Digital Earth, pp. $1-19$.

Koukoulas, S., and Blackburn, G.A., 2001. Introducing New Indices for Accuracy Evaluation of Classified Images Representing Semi-Natural Woodland Environments. Photogrammetric Engineering \& Remote Sensing, 67(4), pp. 499-510.

Laso Bayas, J.C., See, L., Fritz, S., Sturn, T., Perger, C., Dürauer, M., Karner, M., Moorthy, I., Schepaschenko, D., Domian, D., and McCallum, I., 2016. Crowdsourcing in-situ data on land cover and land use using gamification and mobile technology. Remote Sensing, 8(11), 905.

Leyk, S., Uhl, J.H., Balk, D., and Jones, B., 2018. Assessing the accuracy of multi-temporal built-up land layers across ruralurban trajectories in the United States. Remote Sensing of Environment, 204, pp. 898-917.

Liu, C., Frazier, P., and Kumar, L., 2007. Comparative assessment of the measures of thematic classification accuracy. Remote Sensing of Environment, 107, pp. 606-616.

Ma, Z., and Redmond, R.L., 1995. Tau coefficients for accuracy assessment of classification of remote sensing data. Photogrammetric Engineering \& Remote Sensing, 61(4), pp. 435-439.

Manakos, I., Chatzopoulos-Vouzoglanis, K., Petrou, Z.I., Filchev, L., and Apostolakis, A., 2015. Globalland30 mapping capacity of land surface water in Thessaly, Greece. Land, 4(1), pp. 1-18.

Minghini, M., Molinari, M.E., See, L., Fonte, C.C., and Brovelli, M.A., 2017. Preliminary Assessment of the Global Urban Footprint and the Global Human Settlement Layer for the city of Milan. In: Proceedings of the 20th AGILE Conference on Geographic Information Science, Wageningen, The Netherlands.

Mooney, P., and Minghini, M., 2017. A review of OpenStreetMap data. In: Mapping and the Citizen Sensor. Ubiquity Press, London, pp. 37-59.

Mück, M., Klotz, M., and Taubenböck, H., 2017. Validation of the DLR Global Urban Footprint in rural areas: A case study for Burkina Faso. In: Proceedings of the 2017 Joint Urban Remote Sensing Event (JURSE), Dubai, United Arab Emirates.

Neteler, M., Bowman, M.H., Landa, M., and Metz, M., 2012. GRASS GIS: A multi-purpose open source GIS. Environmental Modelling \& Software, 31, pp. 124-130.
Nie, W., Yuan, Y., Kepner, W., Nash, M.S., Jackson, M., and Erickson, C., 2011. Assessing impacts of Landuse and Landcover changes on hydrology for the upper San Pedro watershed. Journal of Hydrology, 407(1-4), pp. 105-114.

Pekel, J.F., Cottam, A., Gorelick, N., and Belward, A.S., 2016. High-resolution mapping of global surface water and its longterm changes. Nature, 540, pp. 418-422.

Pesaresi, M., Ehrlich, D., Ferri, S., Florczyk, A.J., Freire, S., Halkia, M., Julea, A., Kemper, T., Soille, P., and Syrris, V., 2016. Operating procedure for the production of the Global Human Settlement Layer from Landsat data of the epochs 1975, 1990, 2000, and 2014. JRC Technical Report EUR 27741 EN. http://publications.jrc.ec.europa.eu/repository/bitstream/JRC977 05/landsatghs_report_2016_final_online.pdf (30 April 2018).

Pontius, R.G. and Millones, M., 2011. Death to Kappa: birth of quality disagreement and allocation disagreement for accuracy assessment. International Journal of Remote Sensing, 32(15), pp. 4407-4429.

Salk, C.F., Sturn, T., See, L., Fritz, S., and Perger, C., 2016. Assessing quality of volunteer crowdsourcing contributions: lessons from the Cropland Capture game. International Journal of Digital Earth, 9(4), pp. 410-426.

Schultz, M., Voss, J., Auer, M., Carter, S., and Zipf, A., 2017. Open land cover from OpenStreetMap and remote sensing. International Journal of Applied Earth Observation and Geoinformation, 63, pp. 206-213.

See, L., Mooney, P., Foody, G., Bastin, L., Comber, A., Estima, J., Fritz, S., Kerle, N., Jiang, B., Laakso, M., Liu, H.Y., Milčinski, G., Nikšič, M., Painho, M., Pődör, A., Olteanu-Raimond, A.-M., and Rutzinger, M., 2016. Crowdsourcing, Citizen Science or Volunteered Geographic Information? The Current State of Crowdsourced Geographic Information. ISPRS International Journal of Geo-Information, 5(5), 55.

Shimada, M., Takuya, I., Takeshi, M., Manabu, W., Shiraishi, T., Rajesh, T., and Richard, L., 2014. New Global Forest/Non-forest Maps from ALOS PALSAR Data (2007-2010). Remote Sensing of Environment, 155, pp. 13-31.

SPDX, 2018. BSD 3-Clause "New" or "Revised" License. https://spdx.org/licenses/BSD-3-Clause.html (30 April 2018).

Stehman, S.V., 2009. Sampling Designs for Accuracy Assessment of Land Cover. International Journal of Remote Sensing, 30(20), pp. 5243-5272.

Stehman, S.V., Fonte, C.C., Foody, G.M., and See, L., 2018. Using volunteered geographic information (VGI) in designbased statistical inference for area estimation and accuracy assessment of land cover. Remote Sensing of Environment, 212, pp. 47-59.

Thomas, I.L., Benning, V.M., and Ching, N.P., 1987. Classification of remotely sensed images. Adam Hilger, Bristol.

Türk, G., 1979. Gt index: A measure of the success of prediction. Remote Sensing of Environment, 8(1), pp. 65-75.

United Nations, 2015. Transforming our world: The 2030 agenda for sustainable development. A/RES/70/1 https://sustainabledevelopment.un.org/content/documents/21252 $030 \% 20$ Agenda $\% 20$ for $\% 20$ Sustainable $\% 20$ Development $\% 20 \mathrm{w}$ eb.pdf (30 April 2018). 